\title{
Census Division
}

National Cancer Institute

\section{Source}

National Cancer Institute. Census Division. NCI Thesaurus. Code C76337.

Groupings of states that are subdivisions of the four census regions. 